\title{
Open Fracture of Rib
}

National Cancer Institute

\section{Source}

National Cancer Institute. Open Fracture of Rib. NCI Thesaurus. Code C35351.

A traumatic break in one of the bones in the rib cage that involves a break in the adjacent skin. 